\title{
Predictors of intention for continuing volunteer tourism activities among young tourists
}

\begin{abstract}
Volunteer tourism (VT) is an emerging tourism paradigm especially for young tourists. This study inserted three critical constructs (i.e. quality of a VT program, trust in a VT program organization, and awareness of need) into the original theory of planned behavior (TPB) model. Our results indicated that newly-added constructs and the original TPB constructs significantly contribute to the formation of VT tourists' intention for continuing VT activities. Trust, attitude, and subjective norm played vital roles. In addition, awareness was found to be a significant moderator. Overall, our theoretical framework has deepened our understanding of VT tourists' behaviors.
\end{abstract}

Keyword: Volunteer tourism; Young tourists; Theory of planned behaviour; VT destination; Trust; Awareness of need; Quality of a VT program; Intention to continue doing VT activities; Moderating effect; Mediating effect 